\title{
Conocimientos y comportamientos de los usuarios de la red social Facebook relacionados con la privacidad
}

institucional.us.es/ambitos/

Mercedes Durán Segura

Universidad de Sevilla

mduransegura@us.es

\author{
Juan Francisco Mejías Peligro \\ Universidad de Sevilla \\ juanfcomejpel@gmail.com
}

\section{Resumen}

Este estudio examina tres variables relacionadas con la privacidad de la información en la red social Facebook: la preocupación que muestran los usuarios por la privacidad de la información que se publica en la red social, el conocimiento real que tienen sobre la política de privacidad de Facebook y las propias acciones de privacidad que los usuarios realizan para proteger sus datos privados. Asimismo se analiza la existencia de posibles diferencias de género en estas variables. La muestra estuvo compuesta por 190 jóvenes de entre 20 y 35 años de edad, todos ellos usuarios de la red social Facebook.

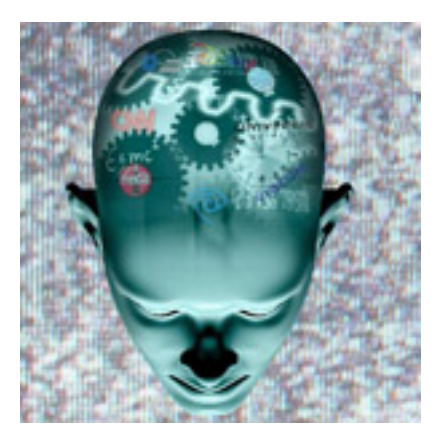

\section{Palabras clave}

Privacidad online, Facebook, información personal, redes sociales, conductas, protección de datos.

\begin{abstract}
This study examines three variables related to the privacy of information in the social network Facebook: the users' concern about the privacy of the information published on the social network, their actual knowledge they have on Facebook's privacy policy and the users' privacy protection behaviors on their private data. Gender differences in these variables were also analyzed. The sample comprised 190 young people aged between 20 and 35 years old. All of them have a Facebook profile.
\end{abstract}

\section{Keywords}

Online privacy, Facebook, personal information, social networking, data protection behaviors.

\section{INTRODUCCIÓN}

Actualmente las redes sociales forman parte del día a día de la mayoría de los jóvenes que encuentran en ellas una forma de relacionarse y de interactuar con otras personas de forma virtual. El tráfico de información anual que registran las redes sociales como Facebook o Tuenti supera al de otras webs como por ejemplo la del INEM o la del Ministerio del Interior (Observatorio Nacional de las Telecomunicaciones, (2012) El simple acceso a estas redes sociales demanda del usuario una serie de datos personales tales como nombre, apellidos o lugar de residencia, por mencionar algunos, a los que se añaden gustos o preferencias personales sobre películas, libros, hobbies, etc... A esta información que se aporta en el momento inicial de creación del perfil se añaden después las redes de amistad (Boyd, 2007), lo que crea una serie de lazos entre los perfiles que permite a las redes sociales sugerir contactos que podrían estar interesados en los mismos temas que el usuario, que han compartido centro de estudios o lugar de procedencia entre otras cosas (Stone, 2008). De esta forma las redes sociales van conformando una nueva realidad relacional entre los usuarios cuyo objetivo fundamental es optimizar las relaciones sociales.

Sin embargo, parece ser que la brevedad temporal que conlleva la aceptación de las condiciones necesarias 
para crear una cuenta y el entusiasmo por poder acceder a los servicios de estas redes sociales hace que la gente no repare en la cantidad de información de tipo personal que voluntariamente se vuelca en las mismas. Desde el ámbito de la Psicología Social interesa conocer si realmente la gente realiza esta cesión gratuita de datos personales sin tener consciencia de ello y sin ningún tipo de crítica. Es decir, qué hace que miles de usuarios regalen información de tipo personal a redes sociales que los venden a otras empresas a un alto precio cuando en otras situaciones habría generado el rechazo de la persona. Este trabajo se interesa por estudiar el conocimiento que tienen los usuarios de la red social Facebook sobre diferentes aspectos relacionados con la privacidad de sus datos, y trata de responder a preguntas como ¿realmente los usuarios conocen las implicaciones del consentimiento que firman al entrar en la red social Facebook? ¿Hasta qué punto el uso cotidiano de esta red consigue normalizar la entrega de datos privados y diluir la conciencia sobre las implicaciones que conllevan esta cesión de información?

La revisión de la literatura realizada pone de manifiesto que, aunque el estudio del comportamiento de los usuarios de las redes sociales es un tema que acapara la atención de los investigadores dentro de la Psicología, la mayoría de estos trabajos se centran en investigar los diferentes usos que se le dan a estas redes sociales o diferentes aspectos relacionados con la personalidad de los usuarios, encontrándose escasa literatura que se haya interesado por el estudio de las creencias o conocimiento por parte de los usuarios sobre el tratamiento de su información personal, siendo además estos estudios realizados con población americana (Sheehan, 1999; Hoy, Mariea Grubbs, Milne y George, 2010).

Estos estudios que acabamos de comentar indagan sobre las creencias, preocupaciones y conductas de protección de los usuarios de la red social Facebook. En términos generales muestran que los usuarios manifiestan cierta preocupación por lo que ocurre con la información privada que entregan a Facebook, sin embargo evidencian un desconocimiento moderado sobre los aspectos relacionados con el tratamiento que hace la red de su información. Estos estudios también destacan diferencias de género en estas variables. Por ejemplo Sheehan (1999) mostró que las mujeres eran más conscientes que los hombres de los riesgos que conlleva aportar información privada a las redes sociales. Otro estudio que también se han interesado por el análisis de las diferencias de género fue llevado a cabo por Hoy y colbs. (2010). Los autores constataron la existencia de diferencias de género en el sentido de que las mujeres mostraban una mayor preocupación por el uso que hacía Facebook de su información privada y llevaban a cabo más medidas que los hombres destinadas a proteger su información privada. Además, no solo realizaban una mayor cantidad de medidas que los hombres, sino que además solían ser acciones diferentes a las emprendidas por los hombres. A la luz de estos resultados se podría afirmar que las mujeres hacen un uso más seguro de las redes sociales ya que muestran una mayor preocupación por ello, están más concienciadas y utilizan diferentes medidas destinadas a proteger su intimidad. Sin embargo, un estudio realizado por Koleck y Saunders (2008) encuentra que las mujeres revelan más información de tipo personal que los hombres en su uso diario de las redes sociales.

Uniendo los hallazgos de ambas investigaciones podriamos llegar a la contradictoria conclusión de que las mujeres realizan más medidas de protección y son más conscientes de los riesgos que presentan las redes sociales, sin embargo en su uso diario revelan más información personal que los hombres.

Este trabajo de investigación replica el estudio realizado en población estadounidense por Hoy y colbs. (2010) en una muestra de población española usuaria de la red social Facebook, con el objetivo de conocer los niveles de preocupación, conocimientos y conductas de prevención llevadas a cabo por los usuarios de la red social Facebook en España. Asimismo, se analizan las diferencias de género en estas variables. En base a las mismas proponemos las siguientes hipótesis:

Hipótesis 1. Los participantes mostrarán niveles altos de preocupación por el tratamiento que la red social Facebook hace de su información privada.

Hipótesis 2. Los participantes mostrarán un nivel moderado de conocimiento sobre el tratamiento que la red social Facebook hace de su información privada.

Hipótesis 3. Los participantes informarán de la realización de conductas dirigidas a la protección de sus datos.

Hipótesis 4. Esperamos encontrar diferencias de género en los niveles de preocupación, conocimientos y 
conductas de protección informadas por los y las participantes en el estudio.

\section{METODOLOGÍA}

\subsection{Participantes}

Participaron en este estudio de manera voluntaria 190 Jóvenes de entre 20 y 35 años de edad, de ambos sexos (68 hombres y 122 mujeres) con una media de edad de 24 años. Todos los y las participantes, que manifestaron ser de nacionalidad española, eran usuarios de la red social Facebook. En cuanto a su nivel educativo, el $93 \%$ eran estudiantes universitarios, el $3 \%$ estaba haciendo un ciclo superior, el $2 \%$ un ciclo medio y el $2 \%$ bachillerato.

\subsection{Instrumentos}

Se diseñó un cuestionario virtual que contenía los siguientes instrumentos y medidas:

- Una primera sección que recogía información sociodemográfica de los participantes: sexo, edad, nacionalidad y nivel académico.

- Escala de Preocupación por la privacidad (Hoy y Milne, 2010). Se trata de una escala compuesta por 7 ítems que preguntan por la preocupación acerca de la privacidad al utilizar la red social Facebook. Las afirmaciones hacen referencia a la preocupación que les puede suscitar a los usuarios lo que ocurre con sus datos una vez son expuestos en la red social, y el tratamiento que esta le da a los mismos. Se responde a través de una escala de respuesta tipo Likert de 5 puntos, siendo 1 (totalmente en desacuerdo con la afirmación) y 5 (totalmente de acuerdo con la afirmación).

- Escala de creencias y conocimiento sobre la privacidad (Hoy y Milne, 2010). La escala contienen 9 afirmaciones sobre el conocimiento y las creencias que los usuarios poseen acerca de lo que ocurre con sus datos. Es decir, a diferencia de la anterior escala que se ocupaba de conocer el grado de preocupación de los usuarios sobre lo que ocurre con los datos, esta segunda sondea el conocimiento real que tienen acerca de lo que ocurre con sus datos. Presenta una escala de respuesta tipo Likert de 5 puntos, donde 1 indica que no tiene consciencia sobre la situación propuesta y 5 es muy consciente. La consistencia interna para esta escala fue de $\alpha=.68$.

- Escala de conductas de privacidad generales (Hoy y Milne, 2010). Esta escala explora en primer lugar si la persona ha leído o no la política de privacidad de Facebook (con respuesta dicotómica). Si la persona contesta afirmativamente evalúa diferentes aspectos con la lectura de la política de privacidad y tiempo dedicado a ello. A continuación indaga sobre conductas generales que pueden realizar los usuarios para proteger su privacidad en la red social, es decir acciones realizadas por el usuario a la hora de usar la red social. La escala de respuesta es de 5 puntos, desde 1 "nunca lo hago" hasta 5 "siempre lo hago". La consistencia interna para esta última categoría de ítems fue de $\alpha=.72$

\subsection{Procedimiento}

La recogida de datos se llevo a cabo de forma virtual, mediante el servicio Google Docs. El enlace de la encuesta se publicó en los muros de Facebook de personas voluntarias y se aprovecho el efecto "bola de nieve" para llegar a nuevos usuarios.

\subsection{Análisis de datos}

Para el análisis de datos se empleó el paquete estadístico SPSS. Se llevaron a cabo comparaciones de medias de cada una de las escalas en función del género utilizando el estadístico t-student. La comparación de medias entre genero se ha llevado a cabo mediante comparaciones de medias de muestras independientes t-test, con corrección de Bonferroni con un nivel de significación $(\alpha=, 01)$.

\section{RESULTADOS}


Analizando las puntuaciones de la muestra total, en general se observa la existencia de niveles medios-altos de preocupación por el tema de la privacidad en las redes sociales. La media obtenida en la escala global $(M=$ 3.53) así lo pone de manifiesto. Las mayores puntuaciones medias de los y las participantes se encuentran en los ítems $1(M=4.14)$, ítem $3(M=4.23)$ e ítem $5(M=4.80)$. Por el contrario, destaca la baja puntuación en el ítem $2(M=2.40)$ que indica la escasa confianza que los usuarios tienen en que Facebook proteja sus datos personales. Por último, y a pesar de que existe una alta preocupación en los usuarios por la privacidad y al mismo tiempo una mínima confianza en la protección que realiza la red, la puntuación en los ítems $5(M=4.80)$ y $6(M=2.83)$ que tratan sobre situaciones en las que se ha podido vulnerar su intimidad es relativamente baja, es decir, les ha ocurrido pocas veces.

Tabla 1. Puntuaciones medias obtenidas por los usuarios de la red social Facebook en la escala de preocupación por la privacidad.

Items

Media

General

1. Me preocupa la privacidad de la información que publico en Facebook

2.Las Redes Sociales hacen un buen trabajo para proteger mi privacidad

3. Es muy importante para mí conocer cómo será usada mi información personal cuando la publico.

4. No sé cómo Facebook puede usar la información que publico en mi perfil.

5. Desearía que me avisaran cada vez que una compañía indaga sobre cómo utilizo

Facebook.

6.En el pasado otras personas han publicado información sobre mí que yo desearía que no se hubiese publicado

7. En el pasado la gente ha visto información en mi perfil que me arrepiento de haber publicado.

Cuando analizamos las puntuaciones de esta escala en función del género no se obtienen diferencias significativas entre hombres $(M=3.47)$ y mujeres $(M=3.56)$, por lo que podemos concluir que tanto hombres como mujeres muestran niveles de preocupación similares por aspectos relacionados con la privacidad en Facebook. A continuación se presentan las medias obtenidas en cada uno de los ítems por hombres y mujeres, y en su caso si esas diferencias son estadísticamente significativas (Tabla 2). Como podemos observar, solo se encuentran diferencias estadísticamente significativas entre hombres y mujeres en el ítem 4, donde las mujeres informan conocer menos el tratamiento que Facebook realiza con sus datos que los hombres.

Tabla 2. Puntuaciones medias, en función del género, obtenidas por los usuarios de la red social Facebook en la escala Preocupación por la privacidad

\begin{tabular}{|l|c|c|c|}
\hline Items & Hombres & Mujeres & $P$ \\
\hline 1. Me preocupa la privacidad de la información que publico en Facebook & 4.14 & 4.23 & 0.8 \\
\hline 2.Las Redes Sociales hacen un buen trabajo para proteger mi privacidad & 2.40 & 2.33 & 0.7 \\
\hline $\begin{array}{l}\text { 3. Es muy importante para mí conocer cómo será usada mi información } \\
\text { personal cuando la publico. }\end{array}$ & 4.23 & 4.34 & 0.07 \\
\hline 4. No sé cómo Facebook puede usar la información que publico en mi perfil. & 3.60 & 3.90 & 0.003 \\
\hline
\end{tabular}


5. Desearía que me avisaran cada vez que una compañía indaga sobre cómo utilizo Facebook.

6.En el pasado otras personas han publicado información sobre mí que yo desearía que no se hubiese publicado

7. En el pasado la gente ha visto información en mi perfil que me arrepiento de haber publicado.

\begin{tabular}{|l|l|l|}
\hline 4.80 & 4.75 & 0.3 \\
\hline 2.83 & 2.90 & 0.4 \\
\hline 2.35 & 2.50 & 0.2 \\
\hline
\end{tabular}

\section{Conocimientos sobre la privacidad}

En cuanto a las creencias y conocimientos que tienen los usuarios sobre la privacidad en Facebook, la media de las puntuaciones obtenida por la muestra $(M=3.08)$ indica un grado medio de conocimiento sobre las acciones que la red realiza para proteger su privacidad. A continuación se pueden ver las medias globales obtenidas por los y las participantes en cada uno de los ítems que conforman la escala (tabla 3).

Tabla 3. Puntuaciones medias obtenidas por los usuarios de la red social Facebook en la escala Conocimientos sobre la privacidad.

Items

1.Los anunciantes rastrean el contenido online que veo

2.Los anunciantes usan la información de mi perfil de Facebook para enviarme anuncios adaptados a mi

3. No me importaría que Facebook permitiera a los anunciantes usar la información de mi perfil

para mandarme anuncios sobre las cosas en las que podría estar interesado/a

4.Las Organizaciones sin ánimo de lucro usan la información de los perfiles como una manera para identificar posibles donadores (contribuyentes)

5.Basado en lo que publico en mi perfil sería difícil para un extraño localizarme en la vida real

6.No me importaría que una persona que pueda contratarme viera lo que publico en mi perfil

7.A veces la gente curiosea la información de otros perfiles como forma de entretenimiento

8.El gobierno usa los perfiles de las redes sociales como una medida para tener control sobre informaciones personales

9. Los profesores tienen en cuenta la información del perfil para decidir sobre las notas de sus alumnos.

Como se puede observar, la puntuación en el ítem 7 indica que los usuarios saben que la gente curiosea la información de los perfiles para entretenerse. Por el contrario, el ítem en el que los participantes obtienen una puntuación media más baja es el ítem 9 , indicando que los usuarios creen que los profesores no toman en cuenta la información que vuelcan en Facebook para decidir sobre sus calificaciones.

Cuando realizamos este análisis en función del género, no se observan diferencias estadísticamente significativas en esta variable entre hombres $(M=3.17)$ y mujeres $(M=3.03)$ (tabla 4$)$.

Tabla 4. Puntuaciones medias, en función del género, obtenidas por los usuarios de la red social Facebook en la escala Conocimiento sobre la privacidad.

Hombres

3.69

Mujeres 3.59
Significación

0.3 
2.Los anunciantes usan la información de mi perfil de Facebook para enviarme anuncios adaptados a mi

3.No me importaría que Facebook permitiera a los anunciantes usar la información de mi perfil para mandarme anuncios sobre las cosas en las que podría estar interesado/a

4.Las Organizaciones sin ánimo de lucro usan la información de los perfiles como una manera para identificar posibles donadores (contribuyentes)

5.Basado en lo que publico en mi perfil sería difícil para un extraño localizarme en la vida real

6.No me importaría que una persona que pueda contratarme viera lo que publico en mi perfil

7.A veces la gente curiosea la información de otros perfiles como forma de entretenimiento

8.El gobierno usa los perfiles de las redes sociales como una medida para tener control sobre informaciones personales

9. Los profesores tienen en cuenta la información del perfil para decidir sobre las notas de sus alumnos.

\begin{tabular}{|l|l|l|}
4.16 & 3.88 & 0.04 \\
2.54 & 2.24 & 0.01 \\
2.64 & 2.51 & 0.8 \\
3.16 & 2.94 & 0.06 \\
2.98 & 2.93 & 0.9 \\
4.65 & 4.86 & 0.000 \\
3.16 & 2.78 & 0.4 \\
\hline 1.57 & 1.57 & 0.2 \\
\hline
\end{tabular}

\section{Conductas de privacidad}

Antes de analizar la escala de conductas generales de protección de la privacidad por parte de los usuarios, se realizaba una primera pregunta a los participantes sobre la lectura de la política de privacidad de la red social Facebook y cuánto tiempo hacía de ello. El $64 \%$ de los participantes afirmaba que nunca la había leído, seguido del $18 \%$ que informaba haberla leído hace más de 6 meses. Por tanto, respecto a esta variable se puede concluir que los usuarios no leen la política de privacidad de Facebook o los que sí lo hacen, lo hacen solamente antes de crear su cuenta. (Figura 1).

Figura 1. Porcentaje de usuarios que habían leído la política de privacidad de la red social Facebook y tiempo desde su lectura.

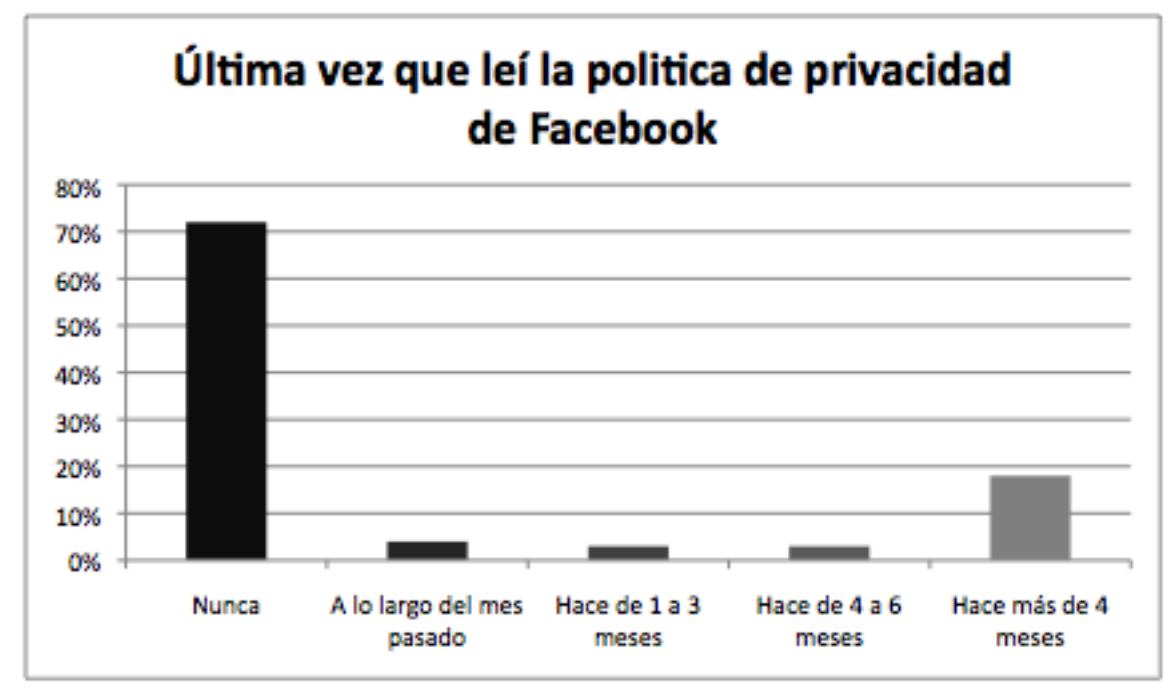

Centrándonos ya en la escala de conductas generales de protección de la privacidad, se obtuvo una puntuación media global de $(M=3.24)$, lo que indica que las conductas de protección se producen de forma habitual entre los/as usuarios/as. A continuación se pueden ver las medias obtenidas en cada uno de los ítems por la muestra global (tabla 5). Analizando esta variable en función del género, no se encuentran diferencias estadísticamente significativas en las puntuaciones medias de hombres y mujeres (tabla 6). 
Tabla 5. Puntuaciones medias obtenidas por los usuarios de la red social Facebook en la escala de Conductas generales de protección de datos.

\begin{tabular}{|l|c|}
\hline Items & $\begin{array}{l}\text { Media } \\
\text { General }\end{array}$ \\
\hline 1.He facilitado información falsa a la hora de crear mi cuenta de Facebook & 2.16 \\
\hline 2.He publicado información personal falsa en mi perfil & 1.75 \\
\hline 3. Reviso regularmente mi configuración de privacidad personal en Facebook & 3.34 \\
\hline 4.Superviso mi perfil de Facebook & 4.25 \\
\hline 5. Soy precavido con las fotos que subo de mí en mi perfil de Facebook & 4.35 \\
\hline 6. Me desetiqueto a mí o a otras personas de fotos en Facebook & 3.30 \\
\hline 7. Soy precavido/a con las personas a la que acepto como amigas. & 4.51 \\
\hline 8. Soy precavido/a con los grupos a los que me suscribo. & 4.09 \\
\hline 9. Me busco en google regularmente. & 2.47 \\
\hline 10. Configuro la privacidad para que mi perfil de Facebook solo sea visible a mis amigos & 4.43 \\
\hline $\begin{array}{l}\text { 11.Controlo mi configuración privada para que lo que hago en Facebook no aparezca en mis } \\
\text { actualizaciones }\end{array}$ & 3.32 \\
\hline 12.Borro mensajes regularmente de mi muro de Facebook & 2 \\
\hline $\begin{array}{l}\text { 13. Uso la nueva configuración de Facebook "lista de amigos" para seleccionar que grupos de } \\
\text { amigos ven diferentes detalles de mi perfil. }\end{array}$ & 2.19 \\
\hline
\end{tabular}

Tabla 6 Puntuación media, en función del género, obtenidas por los usuarios de la red social Facebook en la escala Conductas generales de protección de datos.

\begin{tabular}{|l|c|c|c|}
\hline Items & Hombres & Mujeres & Significación \\
\hline $\begin{array}{l}\text { 1.He facilitado información falsa a la hora de crear mi cuenta de } \\
\text { Facebook }\end{array}$ & 2.31 & 2.09 & 0.34 \\
\hline 2.He publicado información personal falsa en mi perfil & 2.04 & 1.60 & 0.007 \\
\hline $\begin{array}{l}\text { 3. Reviso regularmente mi configuración de privacidad personal en } \\
\text { Facebook }\end{array}$ & 2.88 & 3.60 & 0.59 \\
\hline $\begin{array}{l}\text { 4.Superviso mi perfil de Facebook } \\
\text { 5. Soy precavido con las fotos que subo de mí en mi perfil de Facebook }\end{array}$ & 4.30 & 4.38 & 0.98 \\
\hline $\begin{array}{l}\text { 6. Me desetiqueto a mí o a otras personas de fotos en Facebook } \\
\text { 7. Soy precavido/a con las personas a la que acepto como amigas. }\end{array}$ & 3.16 & 3.37 & 0.52 \\
\hline $\begin{array}{l}\text { 8. Soy precavido/a con los grupos a los que me suscribo. } \\
\text { 9. Me busco en google regularmente. }\end{array}$ & 4.09 & 4.09 & 0.40 \\
\hline $\begin{array}{l}\text { 10. Configuro la privacidad para que mi perfil de Facebook solo sea } \\
\text { visible a mis amigos }\end{array}$ & 4.05 & 4.64 & .000 \\
\hline
\end{tabular}


11.Controlo mi configuración privada para que lo que hago en Facebook no aparezca en mis actualizaciones

12.Borro mensajes regularmente de mi muro de Facebook

13. Uso la nueva configuración de Facebook "lista de amigos" para seleccionar que grupos de amigos ven diferentes detalles de mi perfil.

\begin{tabular}{|c|c|c|}
3.31 & 3.32 & 0.38 \\
\hline 2.16 & 1.7 & 0.05 \\
2.10 & 2.24 & 0.86 \\
\hline
\end{tabular}

\section{DISCUSIÓN}

Karsten Gerloff, presidente de la Fundación de Software Libre de Europa (FSFE), pronosticó que la red social Facebook funcionaría hasta el año 2016 y el motivo principal de esta aseveración lo atribuyó a que Facebook toma a sus usuarios como un producto que vende a sus verdaderos clientes: empresas y servicios secretos. ¿Preocupa esto a la población de jóvenes españoles? Este trabajo se realizó con el objetivo de replicar el estudio de Hoy et al. (2010), realizado en población estadounidense, en una muestra española de usuarios de la red social Facebook. Se analizan, pues, los niveles de preocupación de los usuarios acerca de la privacidad de sus datos en Facebook, su nivel de conocimiento sobre la política de privacidad de dicha red así como las conductas que ponen en práctica tendentes a proteger su privacidad. En cada una de estas variables se analiza la existencia de diferencias de género. En términos generales, los principales resultados de este trabajo replican los hallazgos encontrados por Hoy et al. (2010) en población española.

Respecto a la variable de preocupación, nuestros resultados ponen de manifiesto la existencia de un nivel de preocupación medio-alto entre los jóvenes usuarios españoles acerca del uso que la red social Facebook realiza de su información privada. Este resultado muestra apoyo a la Hipótesis 1 de este trabajo y replica los hallazgos encontrados por Hoy et al., (2010) en esta variable. Ambos estudios muestran que existe un nivel de preocupación medio-alto entre los usuarios de la red, sin embargo en nuestro estudio no se han encontrado diferencias de género en esta variable.

En cuanto al nivel de consciencia y conocimientos que tienen los y las jóvenes acerca del tratamiento que Facebook hace de sus datos, los resultados de este trabajo ponen de manifiesto la existencia de un nivel de conocimiento moderado en los usuarios españoles, mostrando apoyo a la Hipótesis 2 de este trabajo y replicando los hallazgos de Hoy et al. (2010). Al igual que en la anterior variable, y a diferencia de lo encontrado por Hoy et al. (2010) donde los hombres presentan más conocimientos que las mujeres, en este estudio no se han encontrado diferencias de género en esta variable.

Por lo que respecta a las conductas de protección realizadas por los usuarios de Facebook, nuestros resultados muestran que éstas se llevan a cabo de forma frecuente por los usuarios y usuarias de la red, mostrando apoyo a la Hipótesis 3 de este estudio. Tampoco se encontraron diferencias de género en esta variable.

Los principales hallazgos de esa investigación replican el estudio de Hoy et al. (2010) y muestran, además, la no existencia de diferencias de género en estas variables en la población española, lo que no muestra apoyo a la Hipótesis 4 planteada en este estudio. Estos resultados podrían estar reflejando una reducción en la brecha digital de género tradicionalmente existente entre hombres y mujeres tanto en el acceso como en el uso que hacen de las nuevas tecnologías en general, y de las redes sociales en particular. De hecho, este resultado relativo a la reducción de la brecha digital de género ya fue apuntado por el Observatorio de e-igualdad de la Universidad Complutense de Madrid en su último estudio sobre las Redes Sociales. Según los datos de este observatorio, el $82 \%$ de los jóvenes participaban de ellas a menudo y no se detectaron diferencias significativas en su uso por parte de hombres y mujeres (Observatorio de e-igualdad UCM, 2010). Tradicionalmente se ha mostrado cómo las mujeres, en comparación con los hombres, accedían en menor medida a las nuevas tecnologías y además las utilizaban menos, mostrando también un menor conocimiento de ellas. Estos resultados podrían estar reflejando una nueva realidad social en el acceso y utilización de redes sociales donde las diferencias de género habrían desaparecido. Esta es una hipótesis interesante que abre nuevas líneas de investigación para estudios futuros.

Una última cuestión derivada de los resultados obtenidos es la relativa a la discrepancia entre los elevados 
niveles de preocupación que muestran los usuarios y usuarias de la red Facebook, y las acciones concretas destinadas a proteger su privacidad que llevan a cabo estas personas. Esto es, las medidas que llevan a cabo no son tan frecuentes como cabría esperar de una población que se encuentra preocupada por su privacidad en la red. ¿A qué es debido? Como hemos comentado en la introducción, la necesidad del ser humano de socializar e interactuar con otras personas es básica, y parece ser que en la actualidad el "precio" de socializar y no quedar aislado es aceptar los términos y condiciones que dictan las redes sociales. Como se suele decir, "si no estás en la red, no existes".

Este estudio también presenta algunas limitaciones. La primera de ellas hace referencia al tamaño de la muestra utilizada que, en estudios posteriores, debería ser ampliada con el objetivo de poder realizar análisis estadísticos que nos permitan encontrar relaciones entre distintas variables. También sería interesante estudiar estas variables en otros grupos de edad (por ejemplo, adolescentes, adultos) y de diferente nivel educativo, ya que aunque no era nuestro objetivo realizar el estudio exclusivamente con universitarios/as, la mayor parte de los participantes informaba estar cursando estudios universitarios.

En definitiva, los resultados de este estudio replican los de otros trabajos realizados en otros contextos culturales y además evidencian un cambio en los niveles de preocupación, conocimientos y patrones de conducta en hombres y mujeres relativos a la red social Facebook en España. Los hallazgos de este estudio nos invitan a la reflexión y a plantearnos, como psicólogos sociales, la necesidad de diseñar campañas de sensibilización de la población usuaria de las redes sociales, especialmente de los jóvenes que son los principales usuarios, destinadas a hacer más visibles los riesgos que conlleva la entrega de nuestra privacidad a las redes sociales.

\section{REFERENCIAS}

ASCH, S. Fuerzas de grupo en la modificación y distorsión de juicios. En TORREGROSA J.R. Y CRESPO E. (1991) Estudios básicos de la psicología social. Barcelona: Hora (1974) (pp. 351-364).

BEM S. The measurement of psychological androgyny. Journal of consulting and clinical psychology, (1974) $\mathrm{n}^{\circ}$ 42, pp.155-162.

BEM, S. Gender schema theory: a cognitive account of sex typing. Psychological Review, (1981). Nº 88 (4), pp. 354-364.

BOND, R. Y SMITH, P. Culture and conformity: A meta-analysis of studies using Asch's (1952b, 1956) line judgment task. Psychological Bulletin, (1996) n 119. pp 111-137.

CASTILLO, M. R. Y MONTES, B. Escala de estereotipos de género actuales, Iniciación a la investigación, revista electrónica Universidad de Jaén (2007) n 5 pp, 1-21.

FALLOWS, D. How Women and Men Use the Internet. Pew Internet Life Project (2005) in 202-419-4500 http://www.pewinternet.org/

FERNÁNDEZ, J., QUIROGA, M. Á. Y RODRÍGUEZ, A. Escalas de masculinidad y feminidad: estado actual de la cuestión, Psicothema (2007). N 19, pp 357-365.

GARBARINO, E. Y STRAHILEVITZ, M. "Gender Differences in the Perceived Risk of Buying Online and the Effects of Receiving a Site Recommendation" Journal of Business Research, (2004), N 57, pp 768-75.

GOSLING, S. D., AUGUSTINE, A. A., VAZIRE, S. HOLTZMAN, N. Y GADDIS, S. Manifestations of personality in Online Social Networks: self-reported Facebook-related behaviors and observable profile information.

Cyberpsychology, behavior and social networking, (2011) № 14(9), pp 483-8. doi:10.1089/cyber.2010.0087

HOY, M. G. Y MILNE, G. Gender differences in privacy-related measures for young adult facebook users Journal of interactive Advertising (2010). № 10(2), pp 28-45.

KOLEK, E. A. Y SAUNDERS, D. “Online Disclosure: An Empirical Examination of Undergraduate Facebook 
Profiles," NASPA Journal, (2008) n45 (1), pp 1-25, available at http://publications.naspa.org/cgi/viewcontent.cgi? article $=19$ 05\&context=naspajournal (accessed September 10, 2008).

OBSERVATORIO E-IGUALDAD UCM. La brecha digital de género en la juventud Española. Estudio cuantitativo, Publicación digital observatorio de e-igualdad (Diciembre 2010) Universidad Complutense de Madrid. (Madrid). Disponible en www.e-igualdad.net/estadisticas-estudios-igualdad-ucm

PERUGINI, L.M. Y SOLANO, A.C. Análisis de características estereotípicas de género en líderes y seguidores Analysis of gender stereotypic characteristics in leaders and subordinates, SUMMA psicológica UST (2010) $n^{\circ}$ 7(2), 55-66.

MARCUS, B., MACHILEK, F. Y SCHÜTZ, A. Personality in cyberspace: personal Web sites as media for personality expressions and impressions. Journal of personality and social psychology, (2006). $N^{\circ}$ 90(6), pp 1014-31. doi:10.1037/0022-3514.90.6.1014

ONG, E., ANG, R., HO, J. Y LIM, J. Narcissism, extraversion and adolescents' self-presentation on Facebook. Personality and Individual Differences (2011). Retrieved from http://www.sciencedirect.com/science/article/pii/S0191886910004654/

SÁNCHEZ-CARBONELL, X., JORDANA, C. G. Y FARGUES, B. El adolescente ante las tecnologías de la información y la comunicación: internet, móvil y videojuegos. Papeles del Psicólogo, (2007) n² 28(3), pp 196204

SHEEHAN, K. B. "An Investigation of Gender Differences in On-Line Privacy Concerns and Resultant Behavior," Journal of Interactive Marketing, (1999), $\mathrm{n}^{\circ} 13$ (4), pp 24-38

THELWALL, M. Social networks, gender, and friending: An analysis of MySpace member profiles. Journal of the American Society for Information, (2008). № 59(8), pp 1321-1330. doi:10.1002/asi

THOMAS, A. G. Y JOHANSEN, M. K. Inside out: Avatars as an indirect measure of ideal body self-presentation in females. Cyberpsychology: Journal of Psychosocial Research on Cyberspace, (2012). 6(3).

doi:10.5817/CP2012-3-3

WEISBUCH, M., IVCEVIC, Z. Y AMBADY, N. On Being Liked on the Web and in the "Real World": Consistency in First Impressions across Personal Webpages and Spontaneous Behavior. Journal of experimental social psychology, (2009) n 45(3), pp 573-576. doi:10.1016/j.jesp.2008.12.009.

\section{Breve semblanza de los autores}

Mercedes Durán Segura es profesora de Psicología Social en la Facultad de Psicología de la Universidad de Sevilla. Licenciada en Psicología y Doctora en Psicología por la Universidad de Granada, ambos con la obtención de Premio Extraordinario. Realizó dos Máster de especialización, Máster en Psicología de la Intervención Social y Máster en Psicología Jurídica. Actualmente sus intereses de investigación se centran en el estudio de la violencia hacia la mujer, con especial énfasis en el uso de las nuevas tecnologías en el ejercicio de este tipo de violencia, y en los usos y comportamientos relacionados con las redes sociales por parte de los jóvenes.

Juan Francisco Mejías Peligro es Psicólogo y terapeuta en el ámbito de la clínica privada. Licenciado en Psicología por la Universidad de Sevilla. Realizó un Máster de especialización en Práctica Clínica certificado por la European Federation of Psychologist'Associations con el título Europsy, además de cursos de especialización en Psicología Jurídica e Hipnosis clínica. Es miembro de la Unidad de Investigación Psicosocial en Entornos Digitales (UIPED) compuesta por diferentes profesores e investigadores del Departamento de Psicología Social de la Universidad de Sevilla. Sus intereses de investigación se centran en el estudio del comportamiento de los jóvenes en interacción con las redes sociales. 
Ámbitos. Revista Internacional de Comunicación, n.26, año 2014, tercer trimestre (otoño). 